\title{
Principes et problèmes de l'évaluation des résultats en éducation pour la santé
}

\author{
M. Schär, Zürich
}

\section{Résumé ${ }^{1}$}

L'éducation pour la santé se fixe comme but:

1. Faire progresser les connaissances sur la santé physique et psychique, sur les maladies et leurs causes, ainsi que sur les diverses possibilités de prévenir ces maladies.

2. Améliorer les opinions au sujet des problèmes de la santé.

3. Inciter chacun à changer ses habitudes malsaines (nourriture; consommation du tabac, etc.; mode de vie; manque d'activité).

L'accent principal de l'éducation pour la santé à l'école, et surtout pour les adultes, est mis sur la communication par conférences, brochures, articles dans les journaux, radio et télévision. Jusqu'à présent, on ne connait pas exactement la portée de telles actions. Avant tout, on ignore si la communication de ces con-

\footnotetext{
${ }^{1}$ Le texte intégral en langue allemande paraittra dans le no 6/1977 de cette Revue.
}

naissances a un effet durable sur l'attitude et le comportement d'un individu ou de la société.

Pour pouvoir évaluer le progrès de l'éducation pour la santé, il faut faire des études courtes, mais surtout à long terme, correspondant aux bases de l'épidémiologie.

Les groupes de personnes se prêtant aux tests - que décrit exactement le programme d'éducation - doivent être comparées à des groupes témoins qui n'ont pas été éduqués. Le but de telles études doit être non seulement de vérifier l'action immédiate d'une telle mesure, mais aussi de rechercher une action à long terme.

Les difficultés consistent à appliquer exactement les programmes d'éducation, à surveiller les tests et les groupes témoins, à conduire l'observationà long terme, plus particulièrement en recherchant et examinant les groupes de personnes qui se prêtent aux tests ainsi que les groupes témoins.

Ces difficultés rencontrées dans l'évaluation d l'édi:cation pour la santé sont illustrées par des exemples. 